\title{
Using Visual Analytics to Maintain Situation Awareness in Astrophysics
}

\author{
Cecilia R. Aragon ${ }^{1} \quad$ Sarah S. Poon ${ }^{2}$ \\ ${ }^{1}$ Lawrence Berkeley National Laboratory \\ Berkeley, CA 94720
}

\author{
Gregory S. Aldering ${ }^{1}$ \\ ${ }^{2}$ Space Sciences Laboratory \\ Berkeley, CA 94720
}

Rollin C. Thomas ${ }^{1}$

\author{
${ }^{3}$ California Institute of Technology \\ Pasadena, CA 91125
}

\begin{abstract}
We present a novel collaborative visual analytics application for cognitively overloaded users in the astrophysics domain. The system was developed for scientists needing to analyze heterogeneous, complex data under time pressure, and then make predictions and time-critical decisions rapidly and correctly under a constant influx of changing data. The Sunfall Data Taking system utilizes several novel visualization and analysis techniques to enable a team of geographically distributed domain specialists to effectively and remotely maneuver a custom-built instrument under challenging operational conditions. Sunfall Data Taking has been in use for over eighteen months by a major international astrophysics collaboration (the largest data volume supernova search currently in operation), and has substantially improved the operational efficiency of its users. We describe the system design process by an interdisciplinary team, the system architecture, and the results of an informal usability evaluation of the production system by domain experts in the context of Endsley's three levels of situation awareness [1].
\end{abstract}

CR CATEgories: I.3.6 [Computer Graphics]: Methodology and Techniques-Interaction techniques; I.3.8 [Computer Graphics]: Applications-Visual Analytics

KEYWORDS: Data and knowledge visualization, scientific visualization, visual analytics, situation awareness, astrophysics.

\section{INTRODUCTION}

In a growing number of government, business, and scientific domains, individuals must make critical decisions under time pressure while evaluating ever larger and more complex and changing data streams. Examples and guidelines for development of visual analytics applications to enable users such as first responders, pilots, and other decision makers to operate effectively without cognitive overload is a critical research topic. In this paper, we describe a visual analytics application we developed for an international astrophysics collaboration operating custom-built equipment under tight time constraints.

The astronomer's task of observing the heavens with a large, multi-million dollar telescope has many parallels with that of operating a jet aircraft. The astronomer must monitor a large and complex set of operational data while maneuvering the telescope and any attached equipment within particular constraints;

1email: \{CRAragon,GAldering,RCThomas\}@1bl.gov

${ }^{2}$ email: sarahpoon@gmail.com

${ }^{3}$ email: quimby@astro.caltech.edu

IEEE Symposium on Visual Analytics Science and Technology October 21 - 23, Columbus, Ohio, USA

978-1-4244-2935-6/08/\$25.00 @2008 IEEE although there are some safeguards to protect against this, the violation of these constraints could lead to damage of the telescope or its key components. The telescope is frequently located in an inhospitable environment, such as a remote mountaintop, where hypoxia (lack of oxygen) is a constant threat. Adverse weather conditions such as extremely high winds, rain, sleet, or snow, are relatively common occurrences which necessitate closing the dome immediately to prevent damage to delicate optics. Even the rise of the sun is a danger, as equipment could be damaged by sunlight focused by the telescope. Equipment failures such as the telescope dome becoming stuck also occur. Further adding to the operational challenges, observing must necessarily be done at night, in the middle of the lowest ebb of human alertness and cognitive ability.

Astronomical objects rise, move across the sky, and set along arcs which many people have difficulty visualizing. Due to the expense of telescope time, nights are often fully scheduled with an array of astronomical objects, which must be observed in a particular order in a tightly scheduled timetable for maximum scientific benefit. Further, the phase of the moon, upper air turbulence, fog, and changing cloud conditions throughout the night can cause unpredictable variations in the schedule, as certain objects become no longer visible at their appointed time slots.

Astronomers must be able to analyze and evaluate a large amount of data and make cognitively demanding calculations under time pressure, while at the same time being fully aware of changing weather conditions, the approach of daylight and other safety issues. They must focus on individually demanding and precise tasks while maintaining an overall understanding of a large amount of dynamic data affecting the telescope's operation and safety. This need to maintain awareness of an overall situation while evaluating an overwhelming amount of critical and ever-changing information was first documented in the aviation and aerospace industry; it is called situation awareness, and has been well-researched in the aviation and human factors literature since the 1980s [2-4].

However, astronomers usually do not have the pilot's advantage of a sophisticated cockpit design to facilitate their tasks. The software and hardware by which telescopes are operated are usually custom-built, one-of-a-kind systems developed years or decades ago, with clumsy, poorly designed or text-based user interfaces. Further, astronomers may be operating telescopes they are unfamiliar with, or remotely, in a different time zone or under different weather conditions, so that normal human diurnal rhythms or other environmental clues are working against their intuition.

In order to assist astronomers at the Nearby Supernova Factory [5] to maintain situational awareness and perform time-critical science tasks under these conditions, we developed a set of visualization and analysis tools collectively called Sunfall Data Taking. This system is a component of a larger visual analytics system, Sunfall (SuperNova Factory AssembLy Line), that we also developed. It has been in operation since 2006.

Usability studies of pilots and first responders have demonstrated the effectiveness of simplifying visualizations that 
provide key information aligned to human cognitive abilities [6-8]. Adapting such guidelines to the astrophysics domain, our system features a visualization consisting of a mapping of the sky to a rectilinear grid, where astronomical objects move linearly from left to right over time, enabling easy prediction of multiple object movement and visibility throughout the night. Other analysis and visualization tools are also provided to enable the scientists to make appropriate decisions concerning telescope operation and data management rapidly and correctly.

This paper is organized as follows: Section 2 describes the science background for which our system was developed; Section 3 provides a brief introduction to the Sunfall framework. Section 4 discusses related research. Section 5 describes the overall architecture, design, and implementation of Sunfall Data Taking. Section 6 presents a case study and the results of an informal usability evaluation of the production system by astrophysicists using the software to gather and analyze science data. Finally, section 7 offers our conclusions and suggests directions for further work.

\section{SCIENCE BACKGROUND}

One of the grand challenges in astrophysics today is the effort to comprehend the mysterious "dark energy," which accounts for three-quarters of the matter/energy budget of the universe. The existence of dark energy may well require the development of new theories of physics and cosmology. Dark energy acts to accelerate the expansion of the universe (as opposed to gravity, which acts to decelerate the expansion). Our current understanding of dark energy comes primarily from the study of supernovae $[9,10]$.

The Nearby Supernova Factory (SNfactory) [5] is an international astrophysics experiment designed to discover and measure Type Ia supernovae in greater number and detail than has ever been done before. These supernovae are stellar explosions that have a consistent maximum brightness, allowing them to be used as "standard candles" to measure distances to other galaxies and to trace the rate of expansion of the universe and how dark energy affects the structure of the cosmos. The SNfactory receives 50-80 GB of image data per night, which must be processed within 12-24 hours to find potential supernova candidates immediately and obtain maximum scientific benefit from the study of these rare and short-lived stellar events.

Promising supernova candidates are sent for confirmation and spectrophotometric follow-up by SNIFS (the SuperNova Integral Field Spectrograph) [11] on the University of Hawaii $2.2 \mathrm{~m}$ telescope on the summit of Mauna Kea, Hawaii. Candidates are imaged through a $15 \times 15$ microlens array on SNIFS, the spectral data are processed at the summit, and then saved to a remote database.

In order to maximize the efficiency of each night of telescope operation, the scientists put a great deal of effort into parallelizing operations such as telescope slew with data readout, or calibration lamp warm-up with image acquisition. This had the effect of substantially increasing the workload of the person conducting the observations. On a typical night, the SNIFS schedule contains approximately 15 supernova spectra, 7 or 8 standard star spectra, and numerous associated calibration observations (arc lamps for wavelength/instrument flexure mapping and continuums for flux calibration). During each exposure, parallel operations must be conducted while maintaining awareness of the exact moment when the exposure will end so as to evaluate the observed target and make an immediate followup decision. Timing is critical because the schedule is so highly optimized.

It is this portion of the supernova followup pipeline, involving full nights of observation remotely operating the SNIFS spectrograph on the UH $2.2 \mathrm{~m}$ telescope, that Sunfall Data Taking is designed to address. Since the system was put into production, the average number of supernova targets successfully observed each night has increased, and the number of human errors causing missed targets or incomplete schedules has decreased.

Previously, the scientists had believed that the key to solving the problem of human error was to automate the supernova target acquisition process as much as possible, and give the individual at the telescope as little to do as possible. This was reminiscent of descriptions of "pilot error" a few decades ago; pilots operating increasingly complex aircraft systems were making mistakes. When some of these mistakes led to spectacular catastrophes [12], studies were devoted to finding and eliminating the cause of these mistakes. The loss of situation awareness figured prominently in many cases of pilot error [13].

After an evaluation of the scientists' procedures at the telescope, it became evident that the observing environment possessed many of the same characteristics as an aircraft cockpit, and that some of the principles of usability and situational awareness which had been successfully applied in the aviation arena might be adapted to the development of software for the SNfactory astrophysicists.

\section{SUNFALL FrameWORK}

A multi-disciplinary team of computer scientists and astrophysicists was involved in all aspects of the design and implementation of the Sunfall (SuperNova Factory AssembLy Line) software framework for the SNfactory. Sunfall is a collaborative visual analytics system that accelerates science discovery by utilizing novel interactive visualization and analysis techniques to facilitate insight into complex, noisy, highdimensional, high-volume, time-critical scientific data. The system combines sophisticated image processing algorithms, statistical analysis, and machine learning with highly interactive visual interfaces to enable collaborative, user-driven scientific exploration of supernova image and spectral data.

Sunfall is currently in operation at the Nearby Supernova Factory; it is the first visual analytics system in production use at a major astrophysics project.

A major component of the Sunfall framework is the Supernova Warehouse (SNwarehouse), a comprehensive supernova data management, workflow visualization, and collaborative scientific analysis tool. The SNwarehouse contains a PostgreSQL database hosted on a dedicated SNfactory database server running SuSE Linux 9.3, middleware, and a graphical user interface (GUI) implemented in Java. Sunfall Data Taking is the component of SNwarehouse that is specifically designed for use during telescope operation.

Collaboration scientists can access the Sunfall Data Taking GUI from any networked computer worldwide via a remote deployment mechanism. Security is provided via password authentication and encrypted communication channels. SNwarehouse furnishes project scientists with a shared workspace that enables easy distribution, analysis, and access of data. Collaboration members can view, modify, and annotate supernova data, add comments, change a candidate's state, and schedule follow-up observations from work, home, while observing at the telescope, or even when attending conferences. This access is critical due to the 24/7 nature of SNfactory operations. All transactions are recorded in the SNwarehouse database, and the change history and provenance of the data are permanently stored (records cannot be deleted in order to maintain the change history) and continuously visible to all authenticated users.

This paper focuses only on the Sunfall Data Taking component of the system and its simplifying Sky visualization. 


\section{Previous Work}

We discuss related work in the areas of astronomical applications, including calculations and visualizations, and visual analytics systems developed for cognitively loaded or timepressured users. We also briefly touch on scientific analytics and situation awareness research.

The astronomical calculations in the Sky visualization are derived from SkyCalc, originally written by John Thorstensen [14]. Previously, astronomers would run SkyCalc from the command line at the telescope, inputting their observatory parameters, observing date, and target lists. This would provide times of sun and moon rise/set and hourly tables of the elevations of each target. The astronomer would then try to collate this information, but would have to reinput many parameters to examine a slightly different "what if" scenario. Visual wrappers to SkyCalc have been developed before, including Thorstensen's "SkyCalc GUI" and "SkyCalcDisp" [37]; however, the former is simply a graphical version of the command-line interface and the latter contains a three-dimensional projection of object positions that does not facilitate prediction of target motion. On the other hand, the Sky visualization in Sunfall Data Taking was designed specifically to help the astronomer balance the above-described constraints in a visually intuitive way-often during a nighttime observing session-at the click of a button.

The Sky differs from other astronomy visualization programs in that many of them are after-the-fact image processing programs, designed to enhance faint or low-contrast image data, rather than to provide an operational simplification to aid in time-constrained observational decisions $[15,16]$. Astronomers frequently use tools such as SAOImage ds9 [17] for detailed viewing of their images, or IRAF [38] for spectral visualization. However, neither of these is integrated into an observing package as is Sunfall Data Taking.

In the field of scientific visualization for astronomy, software such as Li et al.'s Scalable WIM [18] focuses on the ability to browse a large-scale three-dimensional model of the universe. Again, this technique is not designed for use under time pressure.

In Illuminating the Path, one of the important focus areas laid out for visual analytics research is the development of systems designed for first responders and other cognitively-loaded users [19]. There has been much recent work in this area, including several systems to visualize time-varying data on mobile devices $[6,7,20,21]$, noting the constraints of updating time-critical visual information on a low-resolution device. Other efforts to develop mobile systems for emergency response include the Measured Response project of the Purdue Synthetic Environment for Analysis and Simulation [22].

Related efforts involve approaches to visualizing time-varying geographic data, such as [23], [24], and [25], and Tesone and Goodall have developed a visual analytics technique, "smart aggregation," specifically to aid with situation awareness [26].

There is a large body of literature on situation awareness in general, and a full description of such literature is beyond the scope of this paper. The book by Endsley et al. [4] provides an excellent survey of the work in this area.

Similarly, there is a developing body of work on scientific analytics, or the application of visual analytics to science problems. Examples can be found in [27] and [28], among others.

In general, however, there has not been much work on timecritical visualizations of scientific data, or on the combination of situation awareness enhancement and scientific data, since it is a de facto assumption that scientists must be pondering and analyzing their data in a contemplative fashion in their offices. Our case study of the application of visual analytics to improve situation awareness for astrophysics users appears to be unique.

\section{Sunfall Data TAKING}

\subsection{Design Process}

In order to design an effective collaborative visual analytics system for the SNfactory, we first conducted, in mid-2005, an extensive, two-month evaluation of the existing SNfactory procedures and environment. Data sources used for evaluation included individual interviews, observation of team members performing typical project tasks, review of existing source code, literature reviews, examination of other supernova search projects, and consultation with physicists and computer scientists with relevant experience building similar scientific software systems.

All Sunfall user interfaces were designed and implemented using participatory and iterative design techniques; for example, interactive prototypes were used to evaluate areas where existing interfaces did not support scientists' workflow. Scientists' feedback sometimes led to major redesigns of the interface. The system was implemented from the beginning with this possibility in mind, so that changes were easily made and accepted as an appropriate part of the development process [29].

Participatory design was a natural choice for Sunfall Data Taking, especially given that expert domain knowledge was needed to define the use cases. Scientists were involved in the design process from the start, helping to define the problem space and outlining the necessary functions.

The design of the Data Taking GUI went through several prototypes prior to release, including low fidelity paper prototypes and high fidelity, semi-functional prototypes. Each prototype was evaluated by the scientists, who provided feedback on areas where the interface was confusing or too tedious to use (i.e. too many steps to perform a particular task). Once deployed, we spent several weeks observing the use of the interface, noting any areas where the interface failed to support tasks or was confusing. Informal followup interviews were conducted after such sessions, to gather additional information needed to make incremental improvements (or major changes) to the interface.

\subsection{Telescope Observing Procedures}

Each night of spectral observations with SNIFS at the University of Hawaii $2.2 \mathrm{~m}$ telescope requires a complex set of procedures involving collaboration among a geographically distributed team, and the operation of legacy software telescope controllers driving one-of-a-kind hardware.

The scientist performing the night's observations is known as the "shifter." The shifter may be located anywhere in the world, although SNfactory collaboration members attempt to distribute the operational tasks among time zones so as to minimize nighttime work. Additional members of the team include a telescope operator physically located in the Hilo, Hawaii operations room, and senior scientist experts who may be physically located on the other side of the Earth from the current shifter. At the end of the shift, another scientist evaluates each night's set of observations using a slightly different view of the same data.

\subsubsection{Legacy Software Interface}

The telescope interface is complex, involving a number of shell scripts which must be run from the command line at various times. Functions of the interface include data monitoring as well as telescope control. The scripts interface directly with the telescope and spectrograph device drivers. There are several systems that directly control the telescope.

The telescope control system (TCS) user interface was written using curses, a Unix terminal control library originally developed in 1977 [30]. When TCS was originally written, space was at such a premium on the screen that the developers were forced to use every possible shortcut; for example, the Scottish word "hie" is 
used to describe the state of one of the mirrors when it is in between two positions.

There are also two "directors," which monitor a large amount of information from three CCD cameras, the SNIFS instrument itself, as well as the TCS. These five information streams are piped into two windows, resulting in an extremely rapid flow of information past the eye. The display is filled with blinking and scrolling text, as numeric values are dynamically updated and various internal program status messages rapidly scroll across several text windows (Figure 1). High priority error messages are printed in red to distinguish them from lower priority status messages; however, all the flickering and movement on the screen can be distracting, especially since relatively unimportant status messages are continuously scrolling past the window.

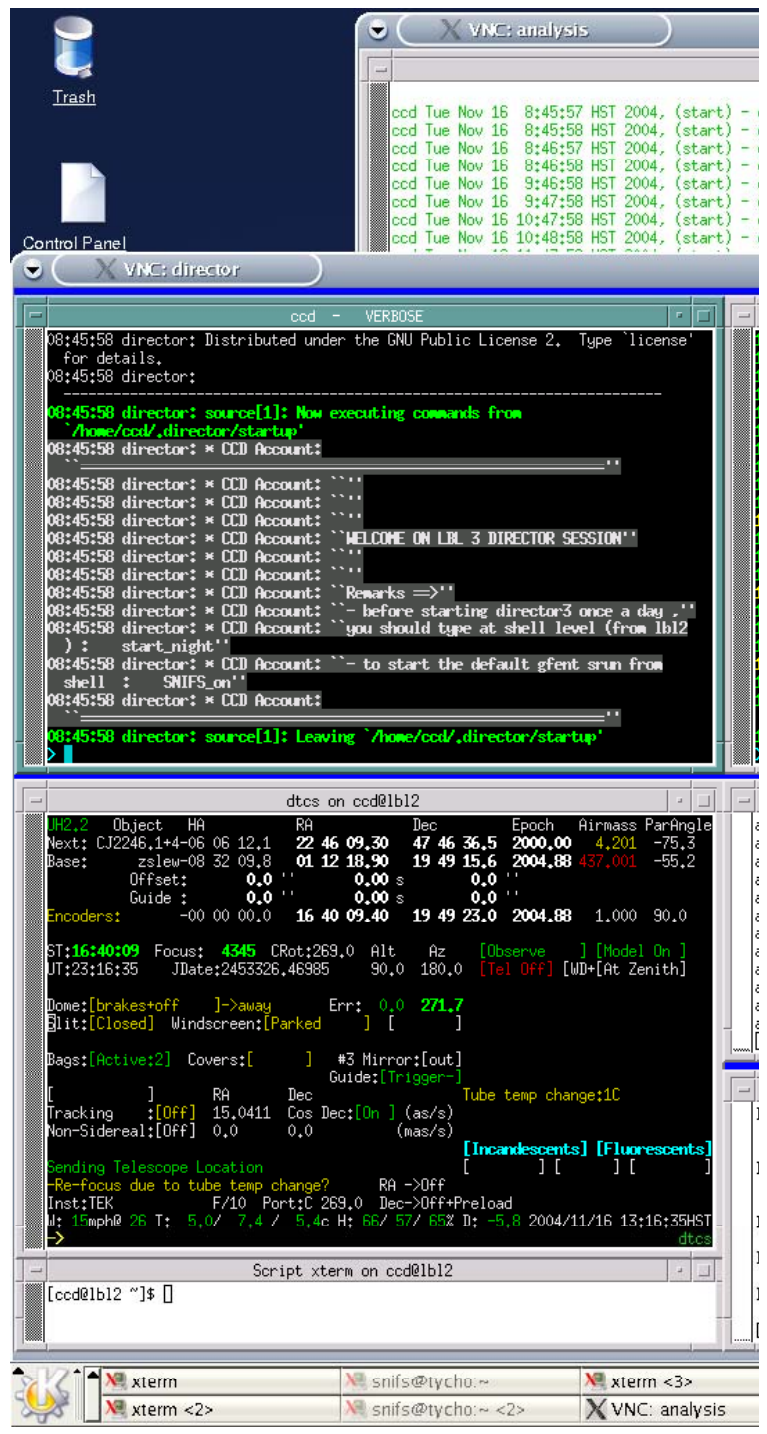

Figure 1. Part of a VNC window of the telescope control system.

To start the night's observing, the shifter must first establish an SSH tunnel to the Mauna Kea summit computer (a Dell Dimension PC running Red Hat Linux), then connect to a VNC (Virtual Network Computing) [31] server on the summit machine, opening two local windows to establish the interface. Two other machines at the summit are custom-built, possessing Tyan motherboards that each enable operation of two camera PCI interfaces simultaneously. The shifter must also connect to a prearranged chat room where all scientists involved in the observation participate, ask questions, provide advice, and use an informal protocol to transfer positive control of telescope operation.

\subsection{Data Taking Development}

To a novice user, the telescope interface is dauntingly complex. The learning curve is extremely steep at the beginning of training. The scientists concluded that it was critical to reduce the shifter's decision-making load. They initially decided that the best method of doing this was to create an "autopilot," an automated instrument control program that would run the entire observing process without human intervention. However, unexpected hardware interactions as well as operational changes ensured that the automated instrument control program could not autonomously run throughout the night. Additionally, it proved impossibly complex to modify the program to reliably compensate for the plethora of unexpected events that could occur during a night's observing; human intervention was consistently required.

Based on this initial experience, it was determined that the shifters needed a decision support system to supplement the automation. Although some level of automation was necessary to reduce drudgery and lighten the shifter's workload, it became clear that a system was needed that combined automation with a visual interface that facilitated human intervention. The scientists instituted various protocol changes and added to the existing system. The Data Taking system was designed and implemented by an interdisciplinary team of computer scientists and astrophysicists, alleviating many sources of shifter error. Tasks the shifters performed with Data Taking included viewing spectral images, exploring data, keeping a standardized "notebook" on sky and instrument conditions, and decision making. The intent was to have the shifters allocate their attention to higher-level tasks; in particular, to evaluating their rate of performance on the schedule, determining whether or not conditions sufficed to perform lowerpriority observations, and assessing data quality.

\subsubsection{Data Taking Components}

The Data Taking GUI is depicted in Figure 2. This interface runs as a separate window on the summit computer and is intended to augment rather than supplement the existing telescope control interface. Operational constraints during software development precluded a complete redesign of the entire telescope control interface, although incremental improvements were made.

The automated instrument control program was left in place; if nothing goes wrong during the night, it will automatically slew the telescope to each target in turn, start an exposure of the correct length, record the data, and move on to the next target. However, in the event of weather or mechanical problems, quick and accurate decision-making is required. Data Taking was designed to support such decisions, with the express goal of improving the shifter's situational awareness. To this end, a number of components were designed to provide supplemental information about critical observing conditions, as well as provide predictive tools to enable the shifter to make scheduling decisions easily and correctly.

Since the shifter is operating the telescope remotely, they may be unaware of deteriorating weather conditions or the approach of sunrise. A software agent running in the chat room broadcasts key astronomical events such as sunrise, and provides information on current telescope operation, including the current exposure or the process of slewing to new coordinates.

The raw data from the SNIFS spectrograph is complex and requires a significant amount of processing to yield meaning to the scientist. A visual depiction of the accuracy of the telescope aim and signal strength provides more information more quickly 
than tables of numeric data. The two small images ("postage stamps") on the left-hand side of each observation subwindow are a custom visualization designed by scientists to indicate whether the telescope is accurately pointed at the target and whether a good signal was received by the spectrograph.

The data taken by SNIFS consists of two channels, blue and red. A channel produces a spectral decomposition of each sample of a $15 \times 15$ pixel image of a 6"x6" square of the sky [11]. Thus, each channel produces an $x$ by $y$ (position) by $\lambda$ (wavelength) "datacube." To make the Data Taking postage stamps, the datacube is collapsed (averaged) along the $\lambda$ axis, leaving an $x$ by $y$ image. Since this image averages over many wavelengths, it is more accurate than a single wavelength slice, roughly as $\left(N_{\text {slices }}\right)^{1 / 2}$, where $N_{\text {slices }}$ is the datacube dimension in the wavelength direction. The program that performs this operation is run as part of the real-time SNIFS pipeline that is run after each spectrum is read out. One of the outputs of this program is the "postage stamp" visualizations, which were integrated into the final version of Data Taking (two images, lower left of Figure 2).

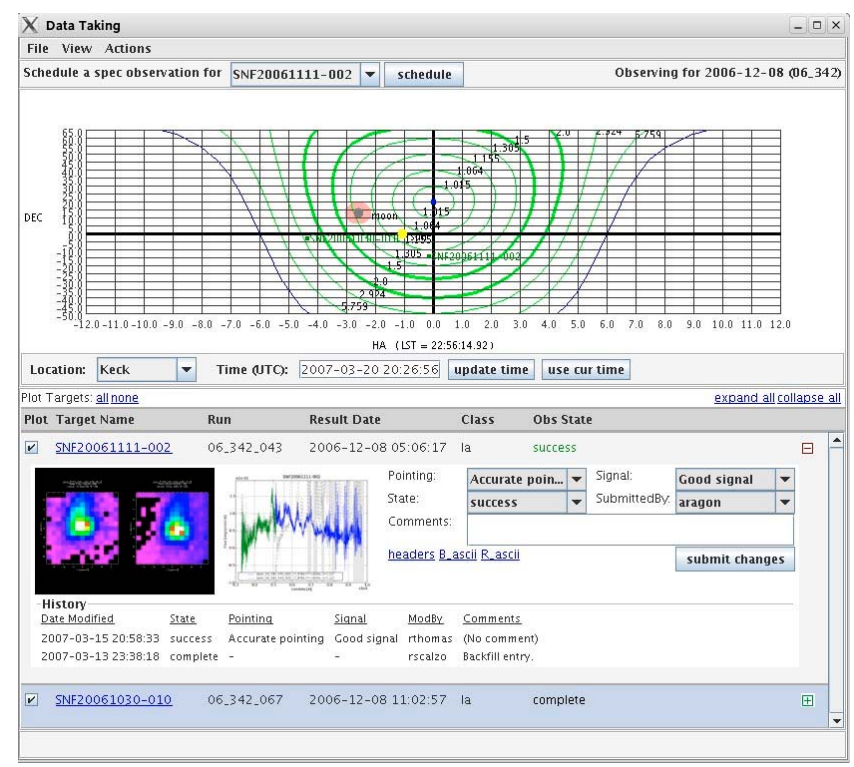

Figure 2. Sunfall Data Taking window. The observer can follow the targets on The Sky visualization, take notes on the success or failure of each observation, telescope status and weather conditions, and can reschedule targets if necessary.

Color-coding and position indicate the accuracy and signal strength of the received data. A bright dot in the center of both postage stamps is a clear indicator of "good centering." A failed observation (due to, say, the passing of a cloud or dome-close trigger due to a humidity spike) contains no such bright dot at the center of the image. A marginal observation may be one where the target is less than optimally centered in the microlens array.

Prior to each night's observation at the telescope, a schedule is made in order to allocate telescope time to each target. In SNwarehouse, the scheduler starts by filtering for only those targets with observation requests. With this list, the scheduler must order and assign exposure times for each target using a variety of techniques. Exposure times are automatically determined using a lookup table based on the phase and redshift of the target. Considering these exposure times, the scheduler must also order the target list according to when the target will be visible in the sky by the telescope. The Sky visualization offers insight for this task (upper section of Figure 2).

\subsection{The Sky Visualization}

It can be challenging for humans to imagine and visualize the path of astronomical objects over time, as they appear to move along the inside of the hemisphere of the night sky. Further, even an astronomer skilled in the art of visualizing celestial coordinates can have difficulty predicting the paths of several objects in the night sky, accurately estimating which of them will avoid clouds, and calculating when they will reach the optimal position for observing.

The key idea in the Sky visualization is the mapping of the spherical night sky to a two-dimensional rectilinear projection, where astronomical objects' paths over time move linearly from left to right. Although there is some spatial distortion (similar to that of a Mercator projection of the Earth [32]), it is much easier for observers to predict the future position of objects moving along a linear path in a plane.

This transformation enables observers to make quick decisions on what targets to observe during the night after unexpected events occur. (Examples of typical changes: new targets come in, clouds increase exposure times, targets are brighter/fainter than expected, instruments fail, etc.).

One of the major causes of changes in the schedule concerns the observation of certain targets called final references. If conditions are not good enough to take a final reference image (a spectrum of the coordinates after the supernova has completely faded), then shifters are supposed to replace those observations with other targets. Additionally, sometimes a screening observation is scheduled (to determine whether a target is even worth following at all), and based on the results, follow-up decisions must be made.

Another use of the visualization involves detecting "packed" parts of the schedule in the Sky. If there are many targets at the same sky coordinates, the shifter knows ahead of time they will have to be efficient -- this appears in the strip chart as a set of targets "stacking up" like books. So, it points to trouble spots requiring extra alertness on the part of the shifter. Thus the Sky offers an intuitive and fast means of adapting to changing conditions and predicting areas of difficulty.

\subsubsection{Sky Visualization Components}

The Sky visualization depicts the positions of targets in the sky at a given time and ground location. The green lines represent airmass (how thick the atmosphere is along the line of sight between an object and the observer on the Earth's surface) for target coordinates at the specified time. The blue line represents the horizon, and the red halo around the moon is the "lunar exclusion zone," the area where light cast by the moon makes it difficult or impossible to view a faint target. The yellow circle represents the sun. Major telescope names and corresponding latitudes and longitudes are displayed on a drop-down menu so the visualization can be applied to other telescopes as well. The time can be changed so the viewer can plan observations for the remainder of the night or even further into the future. If the target appears within the 2.0 airmass lines, it is high enough in the sky to be visible to the telescope, assuming good weather. Airmass is a critical factor in determining quality of astronomical observations. If the airmass increases above 2.0, the quality of the observation drops dramatically.

\subsubsection{Astronomical Calculations and Mapping}

The locations of celestial objects are typically specified in spherical coordinates, right ascension (RA or $\alpha$ ) and declination (DEC or $\delta$ ). RA and DEC are the celestial analogs to longitude and latitude on Earth. The celestial equator is the direct projection of Earth's equator onto the celestial sphere, and defines the reference for declination. The reference for right ascension is the 
First Point of Aries, marked by the location of the sun at the moment of the vernal equinox [33].

Hour angle $(h)$ describes the position of the object based on the local time and gives information about the object's position in the sky relative to a local observer. We map the sky to hour angle vs. declination, so that the observer could see at a glance how many hours an object would be visible, or how long it would be before it rose/set. To do this, the horizon line and lines of constant airmass are mapped onto the displayed rectangle. These can be calculated for any latitude and longitude on the earth. We use:

$$
h=\alpha-L S T
$$

where $h$ is hour angle, $\alpha$ is right ascension, and LST is local sidereal time [14, 33].

Given an observer's longitude and the universal time (UT), one calculates the local sidereal time. Then, given the celestial coordinates of a target $(\alpha, \delta)$, one can calculate the "local" coordinates $(h, \delta)$ used in the Sky.

To calculate the airmass and horizon lines, we determine airmass by approximating the atmosphere as a plane parallel slab. In this approximation airmass equals $\sec (z)$, where $z$ is the zenith distance. Airmass goes to infinity at the horizon (an infinite planeparallel slab).

To draw the lines of constant airmass, including the line that defines the horizon, let $a$ be the altitude, $l$ be the latitude, $\delta$ declination, and $h$ hour angle. Then we have:

$$
\sin (a)=\sin (l) \sin (\delta)+\cos (l) \cos (\delta) \cos (h)
$$

This value is a constant, as these are lines of constant angular distance from the zenith, or equivalently, constant altitude. This allows one to draw a curve on the $(\alpha, \delta)$ plane for any constant altitude, including the horizon, altitude $=0$.

So, for example, to draw the lines at airmass 2.0, we have for any altitude $a$ and zenith distance $z$ :

$$
a=\frac{\pi}{2}-z
$$

and

$$
z=\sec ^{-1}(2.0)=\cos ^{-1}\left(\frac{1}{2.0}\right)
$$

So the airmass 2.0 line is calculated from the above by:

$$
a=\frac{\pi}{2}-\cos ^{-1}\left(\frac{1}{2}\right)
$$

The lunar exclusion zone is defined (where $p$ is the lunar phase, 1 at full moon and 0 at new moon) to be a circle of area $70 p^{2}$ degrees. This is astronomers' generally accepted estimate for how close one might be able to work near the moon, at visible wavelengths, at a good site with clear skies.

\subsection{Other Visualization Tools in Data Taking}

A key component of Data Taking is the access to current and historical supernova spectral data. This information assists scientists to quickly evaluate the priority of a given supernova target compared to other potential targets, when a decision must be made as to which targets to drop from the night's schedule.

Supernova scientists, like many domain experts, demonstrate strong visual pattern recognition ability in their field of expertise. They can take a single glance at a picture of a complex spectrum, and instantly determine its type, age in days before or after peak brightness, and whether it exhibits any unusual properties. An early Type Ia supernova (captured well before peak brightness) will display a certain pattern in its spectral plot. A later supernova (imaged a few days past peak brightness) will show other characteristic spectral lines and features. The spectral data is displayed in optimal form in order to facilitate domain experts' visual pattern recognition ability. Spectral data are plotted in green and blue. The spiky grey lines depict the spectrum of the background sky. The broad grey bands represent areas of atmospheric absorption. Due to the complexity of the data (thousands of points of flux vs. wavelength for each observation) and the necessity to make rapid, accurate decisions in order to maximize the use of limited, expensive telescope time, visualization provides the most efficient solution to the problem. The scientist can also access the raw numeric data by clicking on any of the images or spectra.

\section{INFORMAL USABILITY EVALUATION: AN ASTROPHYSICS Case Study}

The nature of the telescope interface and the high cost of telescope and scientist time precluded formal usability testing of Data Taking. We therefore evaluated Data Taking via informal interviews with scientists who had used the system in the production environment, and by "digital ethnography:" analysis of chat logs recorded during observing sessions at the telescope.

In order to perform this analysis, we evaluated user comments from interviews and chat logs according to five suggested areas for evaluation of visual analytics systems proposed by Scholtz [34]: situation awareness, collaboration, interaction, creativity, and utility, with particular emphasis on situation awareness. Although this analysis is necessarily subjective, it is considered appropriate for operational environments where the user cannot take time out from critical work to answer usability questions.

Endsley has defined situation awareness (SA) as "the perception of elements in the environment within a volume of time and space, the comprehension of their meaning, and the projection of their status in the near future." $[1,4,35]$ Endsley's model defines three stages in the formation of situation awareness: perception (level $1 \mathrm{SA}$ ), or basic monitoring and cue detection of elements in the environment; comprehension (level 2 SA), the ability to interpret this information correctly and combine it with other information; and projection (level 3 SA), the ability to predict what happens next.

The lack of situation awareness has been shown to be a major cause of human error and accidents in many domains [13, 36], especially those with high information flow and potentially serious consequences of errors.

\subsubsection{Procedure}

We conducted interviews with five domain experts, all of whom have been using the telescope control interface for at least two years before Data Taking (DT) was developed and one year after. We also analyzed six months of chat logs from 2007, comprising several thousand user comments from a total of 21 unique users.

\subsubsection{Analysis of User Comments}

We first categorized user comments in the chat logs as positive, negative, or neutral about the Data Taking interface. Although the vast majority of comments were neutral, user comments that expressed an opinion were generally positive (57 positive vs. 13 negative, and the positive comments were much more emphatic than the negative ones). We then further categorized the user comments as follows: general positive comments (39), noticing new features in DT (4), describing DT's utility (5), using DT to make decisions (11), using DT for exploration (2), data processing/image retrieval not completed in a timely fashion (6), lack of transparency in the data processing (2), and limitations (5). We annotated the user comments according to the three levels of SA. These are described below, and examples of each category and how it illustrates the level of SA are presented. 


\subsubsection{Perception: Situation Awareness Level 1}

Level 1 SA, perception, the most basic of the levels of situation awareness, is typically the easiest for a user to develop and a system to support, and often it is taken for granted. However, there were certain cases in our operating environment where it became clear that our system was fostering previously undeveloped perception of objects.

This was demonstrated by the following comments:

"DT forces you to pay attention to standard stars. Before, it was so automatic, it was something that just happened (coffee break time). Yet the standards are so important to calibration, they should pay attention."

"With the new interface, with the targets popping up in the interface, it reminds you to pay attention, you know there are certain pieces of information you need to fill out about each target."

\subsubsection{Comprehension: Situation Awareness Level 2}

At level 2, the operator is synthesizing information to become aware of the big picture, to understand the meaning of the data they are gathering, and to use it to further their own goals. Examples of this are:

"They've actually been looking at the data, the looking is important, $\mathrm{b} / \mathrm{c}$ they wanted to know, what is this guy, what do we know, we see this is the 10th spectrum, it's a nice candidate, people are excited, nobody knows about this guy, what can we find out."

"When they see the spectrum, they become interested in learning more about the target, start looking at the other spectra taken ... they want to know more about this target."

\subsubsection{Projection: Situation Awareness Level 3}

Finally, at level $3 \mathrm{SA}$, the user can accurately predict what it going to happen next. Comments from interviews indicate that DT was quite successful in fostering this final level of situation awareness.

"The Sky allows the shifter to know how [to] prioritize tasks, especially when the schedule changes or falls behind."

"Having a clear view in one plot of where you stand, and how much time you have left to perform the observation of the various target is great... to be 'blind' is much worse."

\subsubsection{Evaluation Areas: Collaboration, Interaction, Creativity, Utility}

Scholtz suggests four other evaluation areas for visual analytic systems: collaboration, interaction, creativity, and utility.

\section{Collaboration}

User feedback indicated that Data Taking enhanced collaboration. Several people remarked on the fact that the tool systematized shifter comments.

"What's great is now every day I get comments about each obs[ervation] from the shifter; [the previous system] didn't do that."

\section{Interaction}

DT greatly enhanced the shifters' ability to interact with the data; this also facilitated their interest and attention, and increased their levels of situation awareness.

"DT gives the shifter data that either they couldn't or was too difficult to access, and now, the shifters are much more interested in exploring the targets while on shift."

Utility

Over and over again, users commented on how useful the program was. An interesting side effect of this is that the utility of the program was perceived as having a calming effect on the shifter. Perhaps this reveals how much an interface with poor usability can add to stress and cognitive load. The scientists also commented on how DT improved everyone's efficiency.

"Having a visual view of the schedule / candidates in this sky image has been extremely useful. Also, and this is one of my main remarks, taking data is hard [on] your nerves ... and when you are nervous ...you make lots of mistake[s] / bad choice[s]"

"In 2007 we had really good efficiency; users [made] minimal mistakes ... and on this point [DT] helped a lot by 'cooling down' the shifter."

"The quick visualization of spectra and reconstructed images is also extremely useful."

"To be brief DT changed my life..."

\section{Creativity: Discovery and Decision-Making}

Creativity is not normally a characteristic one would consider in developing software. However, given the challenging nature of the observing shift, creative solutions to problems are frequently necessary. Thus, we categorized effective decision making and discovery under creativity. User comments indicated that Data Taking facilitated decision-making.

"Having access to the full SNfactory target history ... this helped me a lot ... to point out problem[s] on a specific target (no way we can follow this guy with SNIFS ... make sure that it will not be put in a schedule again ...)"

"Yes, DT has been very useful during my shifts. The graphical view of the objects on the sky ... eased the decision of canceling an exposure or to insert an extra observation in the schedule. I didn't $[\mathrm{m}]$ ake such decisions before DT."

\subsubsection{Discussion and Summary}

In general, Sunfall Data Taking received very positive user feedback. In all five of Scholtz's areas of evaluation, Data Taking received favorable reviews. However, negative comments indicated directions for future work. The background mechanism (Data Forklift) which handled the data processing and image retrieval was sometimes slow or it was unclear what stage of processing the data had reached. This was due in part to the necessary reliance on outside services such as the database in France. This was one area in which scientists' situation awareness could be enhanced further. In future versions, we will include a status window describing the current state of the data transfer and processing.

However, in taking the user comments as a whole, we found evidence that Data Taking has improved scientists' situation awareness, increased overall efficiency, and has assisted scientists to make collaborative decisions. Selective presentation of data, the ability to interact with the data, and the use of simplifying visualizations were all effective in increasing situation awareness in this time-critical scientific application.

\section{CONCLUSIONS AND FUtURE WORK}

We described a case study involving a visual analytics system developed for astrophysicists collaboratively operating a large telescope for time-critical supernova observation. Additionally, we showed the effectiveness of a simplifying visualization, projecting three-dimensional data to a rectilinear two-dimensional format, in increasing situation awareness for users needing to synthesize large amounts of streaming data and make critical decisions under time pressure. This confirms previous studies [68 ] on the value of simplifying visualizations in interfaces designed for cognitively-overloaded users.

Furthermore, we have demonstrated that the cultivation and maintenance of users' situation awareness, a concept first studied in the field of aviation and cockpit management, is crucial in other fields where time-critical decisions involving large, dynamic data sets must be made. 
Finally, perhaps one of the most important lessons to be learned from this case study is the importance of forming closely integrated, interdisciplinary teams where members from all fields are involved in the participatory design process from the beginning of the project. Designing effective, usable interfaces for complex, time-critical scientific decision making requires both domain expertise and software expertise, and the two must be closely intertwined for the ultimate success of the project. In our case, the physicists attributed the successful adoption and general satisfaction with the interface to the participatory design process. The use of this technique allowed the interface to closely match their needs and led to a general sense of ownership of all the software, not just the science algorithms. In addition, all members of the team participated in the software development process, which included regular group meetings and informal code reviews, thus facilitating the marriage of sophisticated science algorithms with software engineering best practices.

Although the users have indicated high satisfaction with the system, certain parts of the user interface can be improved further. For example, when there are many targets displayed in the Sky visualization, their labels overlap and occlude one another. In a future version, we plan to implement excentric labeling as described by Fekete and Plaisant in [39]. Additionally, we plan to include a data processing status window to alleviate some of the difficulties discussed in section 6.1.3. Finally, we plan to address some of the user comments on system limitations, such as adding the ability to interactively change the Data Taking schedule, adjust the DT window size, and recover more smoothly when the middleware fails.

\section{ACKNOWLEDGMENTS}

We would like to thank the anonymous reviewers for their thoughtful suggestions, and the scientists of the SNfactory collaboration for their time and detailed feedback. The authors wish to recognize and acknowledge the very significant cultural role and reverence that the summit of Mauna Kea has always had within the indigenous Hawaiian community. We are most fortunate to have the opportunity to conduct observations from this mountain. This work was supported in part by the Director, Office of Science, Office of Advanced Scientific Computing Research, of the U.S. Department of Energy under Contract No. DE-AC02-05CH11231, and by the Director, Office of Science, Office of High Energy Physics, of the U.S. Department of Energy under Contract No. DE-FG02-92ER40704, and by a grant from the Gordon \& Betty Moore Foundation. This research used resources of the National Energy Research Scientific Computing Center, which is supported by the Office of Science of the U.S. Department of Energy under Contract No. DE-AC02-05CH11231.

\section{REFERENCES}

[1] M. R. Endsley, "Toward a theory of situation awareness in dynamic systems," Human Factors, vol. 37, pp. 32-64, 1995.

[2] M. R. Endsley, "Designing for Situation Awareness in Complex Systems," Proceedings of the Second International Workshop on Symbiosis of Humans, Artifacts and Environment, Kyoto, Japan, 2001.

[3] M. R. Endsley, "Situation Awareness and Human Error: Designing to Support Human Performance," High Consequence Systems Surety Conference, Albuquerque, NM, 1999

[4] M. R. Endsley and D. J. Garland, Situation Awareness Analysis and Measurement: Lawrence Erlbaum Associates, 2000.

[5] G. Aldering, G. Adam, P. Antilogus, P. Astier, R. Bacon, et al., "Overview of the Nearby Supernova Factory," Proceedings of the SPIE, 2002

[6] L. Chittaro, F. Zuliani, and E. Carchietti, "Mobile Devices in Emergency Medical Services: User Evaluation of a PDA-Based Interface for Ambulance Run Reporting," in Mobile Response, vol. 4458/2007: Springer Berlin / Heidelberg, 2007, pp. 19-28.

[7] S. Kim, Y. Jang, A. Mellema, D. Ebert, and T. Collins, "Visual Analytics on Mobile Devices for Emergency Response," IEEE Symposium on Visual Analytics Science and Technology, Sacramento, CA, 2007.
[8] C. Aragon and M. Hearst, "Improving Aviation Safety with Information Visualization: A Flight Simulation Study," CHI 2005: ACM Conference on Human Factors in Computing Systems, Portland, OR, 2005.

[9] S. Perlmutter, G. Aldering, G. Goldhaber, et al., "Measurements of Omega and Lambda from 42 High-Redshift Supernovae," Astrophysical Journal, vol. 1999, pp. 565-586, 1999.

[10] A. G. Riess, A. V. Filippenko, et al., "Observational Evidence from Supernovae for an Accelerating Universe and a Cosmological Constant," Astrophysical Journal, vol. 1998, pp. 1009-1038, 1998.

[11] B. Lantz, et al., "SNIFS: a wideband integral field spectrograph with microlens arrays," SPIE, 2004

[12] A. Degani, Taming Hal: Designing Interfaces Beyond 2001. New York, NY: Palgrave Macmillan, 2004

[13] C. Hartel, K. Smith, and C. Prince, "Defining aircrew coordination: searching mishaps for meaning," 6th Int'l Symposium on Aviation Psychology, Columbus, $\mathrm{OH}, 1991$

[14] J. Thorstensen, SkyCalc User's Manual, http://zimmer.csufresno.edu/ $\sim$ fringwal/skycal.pdf, 1994, accessed 2008.

[15] F. Cavicchio, Astroart 4.0 - 96 bit image processing, http://www.msbastroart.com/, 2008, accessed 2008.

[16] L. Christensen, ESA/ESO/NASA Photoshop FITS Liberator, http://www.spacetelescope.org/projects/fits_liberator/index.html, 2008, accessed 2008.

[17] W. Joye and E. Mandel, "New Features of SAOImage DS9," Astronomical Data Analysis Software and Systems XII, vol. 295, 2003.

[18] Y. Li, C. Fu, and A. Hanson, "Scalable WIM: Effective Exploration in LargeScale Astrophysical Environments," IEEE Transactions on Visualization and Computer Graphics, vol. 12, pp. 1005-1011, 2006.

[19] J. J. Thomas and K. A. Cook, Illuminating the Path: The Research and Development Agenda for Visual Analytics: National Visualization and Analytics Center, 2005

[20] A. Pattath, B. Bue, Y. Jang, D. Ebert, X. Zhong, A. Ault, and E. Coyle, "Interactive Visualization and Analysis of Network and Sensor Data on Mobile Devices," Proceedings of IEEE Symposium on Visual Analytics Science and Technology, Baltimore, MD, 2006.

[21] S. Eick, M. Eick, J. Fugitt, B. Horst, M. Khailo, and R. Lankenau, "Thin Client Visualization," IEEE VAST, Sacramento, CA, 2007.

[22] Purdue, Synthetic Environment for Analysis and Simulation, http://www.mgmt.purdue.edu/centers/perc/html, 2008, accessed 2008.

[23] T. Kapler and W. Wright, "Geotime information visualization," Proceedings of IEEE Symposium on Information Visualization, 2004.

[24] C. Pan and P. Mitra, "FemaRepViz: Automatic Extraction and Geo-Temporal Visualization of FEMA National Situation Updates," IEEE VAST, Sacramento, CA, 2007.

[25] RSOE, RSOE HAVARIA AlertMap, http://hisz.rsoe.hu/alertmap/ woalert.php?lang=eng, 2008, accessed 2008 .

[26] D. Tesone and J. Goodall, "Balancing Interactive Data Management of Massive Data with Situational Awareness through Smart Aggregation," Proceedings of the IEEE Symposium on Visual Analytics Science and Technology, Sacramento, CA, 2007.

[27] C. D. Shaw, G. Dasch, and M. Eremeeva, "IMAS: The Interactive Multigenomic Analysis System," IEEE VAST, Sacramento, CA, 2007.

[28] R. Maciejewski, B. Tyner, Y. Jang, C. Zheng, R. Nehme, and D. Ebert, "LAHVA: Linked Animal-Human Health Visual Analytics," IEEE VAST, 2007.

[29] C. Aragon and S. Poon, "The Impact of Usability on Supernova Discovery," Workshop on Increasing the Impact of Usability Work in Software Development, CHI 2007: ACM Conference on Human Factors in Computing Systems, San Jose, CA, 2007.

[30] K. Arnold, "Screen Updating and Cursor Movement Optimization: A Library Package," University of California, Berkeley 1977.

[31] B. Nguyen, VNC: Virtual Network Computing, http://www.tldp.org/ LDP/Linux-Dictionary/html/index.html, 2004, accessed 2008.

[32] R. Israel, Mercator's Projection, http://www.math.ubc.ca/ israel/ $\mathrm{m} 103 /$ mercator/mercator.html, 2003, accessed 2008.

[33] H. Karttunen, P. Kroger, H. Oja, M. Poutanen, and K. Donner, Fundamental Astronomy, Second Edition ed: Springer-Verlag, 1994.

[34] J. Scholtz, "Beyond Usability: Evaluation Aspects of Visual Analytic Environments," IEEE Symposium on Visual Analytics Science and Technology, Baltimore, MD, 2006.

[35] M. R. Endsley, "Situation awareness global assessment technique (SAGAT)," Proceedings of the National Aerospace and Electronics Conference, 1988.

[36] D. Merket, M. Bergondy, and H. Cuevas-Mesa, "Making sense out of teamwork errors in complex environments," 18th Annual Industrial/Organizational Behavior Conference, Roanoke, VA, 1997.

[37] J. Thorstensen, "SkyCalc GUI Manual," http://mdm.kpno.noao.edu/ Manuals/doc/guimanual.html, accessed 2008.

[38] T. Boroson, et al., "NOAO Image Reduction and Analysis Facility," http://iraf.noao.edu/iraf/web, accessed 2008.

[39] J. Fekete and C. Plaisant, "Excentric Labeling: Dynamic Neighborhood Labeling for Data Visualization," Proceedings of CHI '99, Pittsburgh, PA, 1999 